\title{
The concepts of thresholds of ischaemia in relation to brain structure and function
}

\author{
LINDSAY SYMON, N. M. BRANSTON, A. J. STRONG, AND T. D. HOPE \\ From the Gough Cooper Department of Neurological Surgery, Institute of Neurology, and \\ The National Hospital, Queen Square, London
}

The relationship between the blood supply of a neurone and its capacity to function and survive remains of fundamental importance in the study of cerebral vascular disease from both the surgical and the medical standpoint. Thus it has been assumed that a matter of only a few minutes is necessary to cause complete and permanent loss of function of key neurones in the primate nervous system (Dennis and Kabat, 1939; Weinberger et al, 1940; Grenell, 1946; Boyd and Connolly, 1962), but everyday clinical experience confirms that dense neurological deficits following soon after a cerebral ischaemic episode may gradually resolve and finally even disappear. It has long been an intriguing clinical question whether such resolution of neurological deficits relates to the recovery of neurones so damaged following the ischaemic episode as to cease functioning, yet subsequently recover, or whether associated areas outwith the zone of immediate ischaemia are capable of assuming the function of structures rendered irreversibly damaged by the ischaemic event. The theory that certain cells probably survive in a state of structural integrity but functional paralysis has seemed to us for some years most likely, while possible explanations for the recovery of neurones have been either improvement in residual circulation with expansion of the collateral vessels from neighbouring cerebrovascular beds, or modification of the neuronal metabolism itself so that function may be resumed at a lower basal level of blood flow. The experimental findings in this paper relate attempts to elucidate the relationship between structure, function and blood supply in the nervous system. They have been stimulated by surgical rather than medical phenomena, since the occlusion of vessels during the excision of basal tumours, or the occlusion of portions of the cerebral circulation in the treatment of intracranial aneurysm, generally gives a human preparation less clouded by incidental vascular disease than occlusion in the course of the develop- ment of atherosclerosis with its attendant impairment of function of collateral vessels, and, possibly, disorder reactivity of the vascular system as a whole.

In the experimental analysis of these phenomena the experimental stroke model described elsewhere has been used (Symon et al, 1971; Symon, 1975), in which the middle cerebral artery is occluded either by an intracranial approach along the sphenoidal wing, or by a transorbital approach after removal of the contents of the orbit and dissection through the enlarged superior orbital fissure. Temporary occlusion of the vessel in acute experiments is performed with a small spring clip such as a Scoville clip, while for permanent occlusion the artery is divided between two small 'haemo' clips. The site of occlusion used in this model is the first millimetre of the middle cerebral which, as Shellshear (1921) and Abbie (1934) have indicated, is free from perforating vessels. The intensity of ischaemia in the basal ganglia may be increased by simultaneous occlusion of the perforating vessels.

The distribution of the middle cerebral artery in the experimental primate

Perfusion studies following acute experimental occlusion of the middle cerebral artery (Symon, 1961) and pathological studies of the brain in animals which have survived up to three years following middle cerebral occlusion (Brierley and Symon, 1977) have shown that the average perfusion of the middle cerebral artery in the monkey is very similar to the description of Shellshear (1921) and Abbie (1934) in man. Thus Symon (1961) showed by a technique of carmine-gelatine perfusion that the intensity of perfusion after middle cerebral artery occlusion was reduced in the parietal and temporal lobes, particularly the opercular and insular areas. It was diminished in the basal ganglia and non-perfusion of areas of basal ganglia could be produced by separate occlusion of perforating branches. Disruption of both 
the medial and lateral striate groups of perforating branches in the primate resulted in non-perfusion in the body and caudal part of the head of the caudate nucleus and in the putamen of the lentiform nucleus. The rostral part of the head of the caudate nucleus was perfused from perforating branches of the anterior cerebral artery. In the experimental model also there was good perfusion of the medial part of the globus pallidus, the tail of the caudate nucleus, and incomplete perfusion of the amygdala from branches of the anterior choroidal artery, which, in the primate as in man, arises from the internal carotid artery separately from the middle cerebral artery.

\section{The clinical picture of the chronic stroke model}

As has been extensively reported (Thompson and Smith, 1951; Harvey and Rasmussen, 1951; Sundt and Waltz, 1966; Symon et al, 1975), the technique of acute middle cerebral occlusion with involvement of the perforating vassels produces a clinically recognizable stroke in the large experimental primate. Facial weakness, a semi-flexed resting position of the elbow, wrist and fingers, and impairment of joint position sense obvious in placing reactions closely parallel features produced by classical stroke either in degenerative vascular disease or in middle cerebral arterial stenosis associated with aneurysmal surgery. The rapidity of recovery of elbow and finger movements and of placing reactions is appreciably greater in animals in which perforating damage has been avoided altogether, and it is possible to grade the deficit from virtually undetectable weakness, the hemiparesis appearing only when the animal feeds as a facial weakness, or when it attempts to grasp objects alternately with either hand when the placing reactions can be demonstrated as significantly abnormal, to a moderately dense faciobrachial weakness. The location of the infarct as demonstrated by Dr Brierley involves the basal ganglia, the lips of the Sylvian fissure (the opercula and the insula) while the cortex of the lateral aspect of the brain, apart from the immediate temporal pole, would pass for normal.

The study of the acute experimental primate stroke therefore indicates that soon after the occlusion of the middle cerebral artery detectable loss of function in the face and arm area of the cortex in the ipsilateral hemisphere is evident, and if the infarction is allowed to proceed to maturity, structural loss occurs in the insula, the lips of the Sylvian fissure and the basal ganglia together with an appreciable area of white matter of the centrum semiovale and internal capsule.

\section{Methodological considerations}

DETERMINATION OF THE DISTRIBUTION AND 을 DENSITY OF ISCHAEMIA IN THE STROKE MODEL

The basic method of determination of brain bloog flow used in these experiments has been a technique of saturation and elution of hydrogen gas (Pasztor $e \bar{\phi}$ $a l, 1973)$. This follows the basic principle of inert gas clearance and produces data from the experimentak̂s laboratory which are closely comparable to those $\overrightarrow{0}$ obtained from man by techniques of inert gasclearance such as the use of Xenon 133. The advans. tage of the hydrogen gas technique in the experi mental animal is that the determinations may be made over very small areas of brain, with electrodesc of $300 \mu$ diameter, the area of response being within $a-$ radius of $2 \mathrm{~mm}$, whereas even with the best collimation available, the inert radioactive gas sampling $\vec{P}$ technique involves much larger areas. In the smal

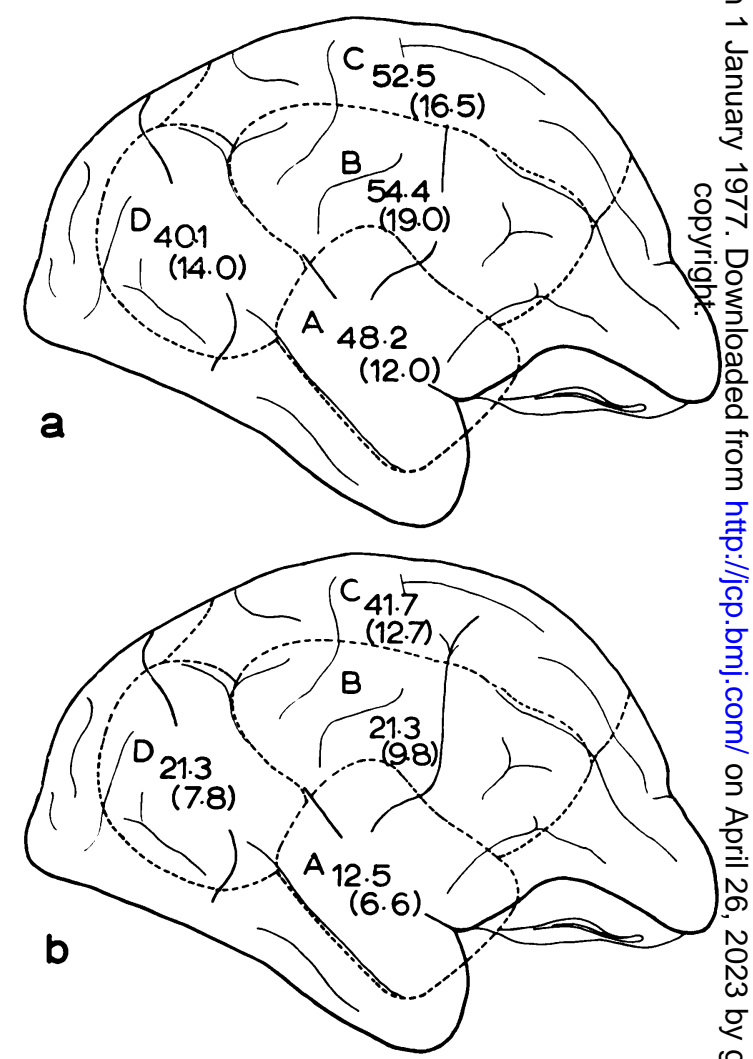

Fig 1a Blood flow values in $\mathrm{ml} / 100 \mathrm{~g} / \mathrm{min}$ with standard $\stackrel{0}{\circ}$ deviation in 11 animals in four areas of normal primate cortex. Flows are at 2 minutes' clearance and averaged

over a number of electrodes in the appropriate zone.
$\mathrm{b}$ Blood flow values in $\mathrm{ml} / 100 \mathrm{~g} / \mathrm{min}$ immediately after occlusion of the middle cerebral artery in 11 baboons. 
animal brain this constitutes a disadvantage. Basic principles of the technique have been described and the standard physiological responses of primate cortical vasculature to such stimuli as hypertension and hypocapnia categorized (Symon et al, 1973). In relation to middle cerebral occlusion, the distribution and density of reduced flow following the middle cerebral occlusion has been mapped out using multiple electrodes, and the findings are shown in (fig 1 compared with the control hemisphere. It is evident that in the region of the Sylvian opercula and also in the basal ganglia, reduction of flow to some 20 per cent of normal is present. In the most anterior portions of the Sylvian opercular lips, this reduction in flow falls to values of $10 \mathrm{ml} / 100 \mathrm{~g} / \mathrm{min}$, in absolute terms, or less. There is a graduated reduction in flow which decreases in intensity as the midline and parasagittal areas are approached. Even in the immediate parasagittal zone, however, flow is affected though the reductions in our experiments did not reach significant levels. In contrast to experiments using detector techniques for arterial blood flow in the pia such as direct thermistor recording, no actual increases in tissue blood flow have been observed in relation to acute arterial occlusion. The sensitivity of the basal nuclei was comparable to that of cortex in the most densely ischaemic zone.

\section{ASSESSMENT OF FUNCTION BY AVERAGED}

\section{CORTICAL EVOKED RESPONSES}

The characteristic pattern of ischaemia following middle cerebral occlusion which has been described (Branston et al, 1974) enabled us to assess the effect of varying degrees of ischaemia on the averaged cortical evoked response. This was produced by stimulation of the contralateral mandibular division of the fifth nerve, the evoked responses being recorded by a plate electrode on the postrolandic cortex in an area which was normally subjected to an intermediate degree of ischaemia following middle cerebral occlusion. The loss of autoregulation which follows middle cerebral occlusion (Symon et al, 1976) enabled us to increase the density of ischaemia in this intermediate zone at will by the induction of systemic hypotension. Flow could be recorded in the immediate area of the evoked response electrode and the relationship of the cortical electrical activity to local perfusion determined. It is possible to do this since the relays immediately preceding the cortex, in the ventro-lateral nucleus of the thalamus, lie, in the baboon, in an area supplied by the choroidal vessels, outwith the distribution of the middle cerebral artery and unaffected by middle cerebral occlusion. The rapidity of changes in the electrical responses induced by appropriate degrees of ischaemia indicate their relation to grey matter function rather than to the much more slowly failing conduction in the white matter. The results of these experiments indicated a threshold relationship between regional blood flow and the somatosensory evoked response. Thus down to levels of blood flow of $20 \mathrm{ml} / 100 \mathrm{~g} / \mathrm{min}$ the evoked response was fully sustained, while at levels below $12 \mathrm{ml} / 100 \mathrm{~g} / \mathrm{min}$ the response substantially disappeared. In the area of 14 to $16 \mathrm{ml} / 100 \mathrm{~g} / \mathrm{min}$ there was a very sharp decline in the evoked response, indicating a threshold relationship at about this level (fig 2). Similar findings have been reported by Boysen et al (1973) in Scandinavia, who have assessed the

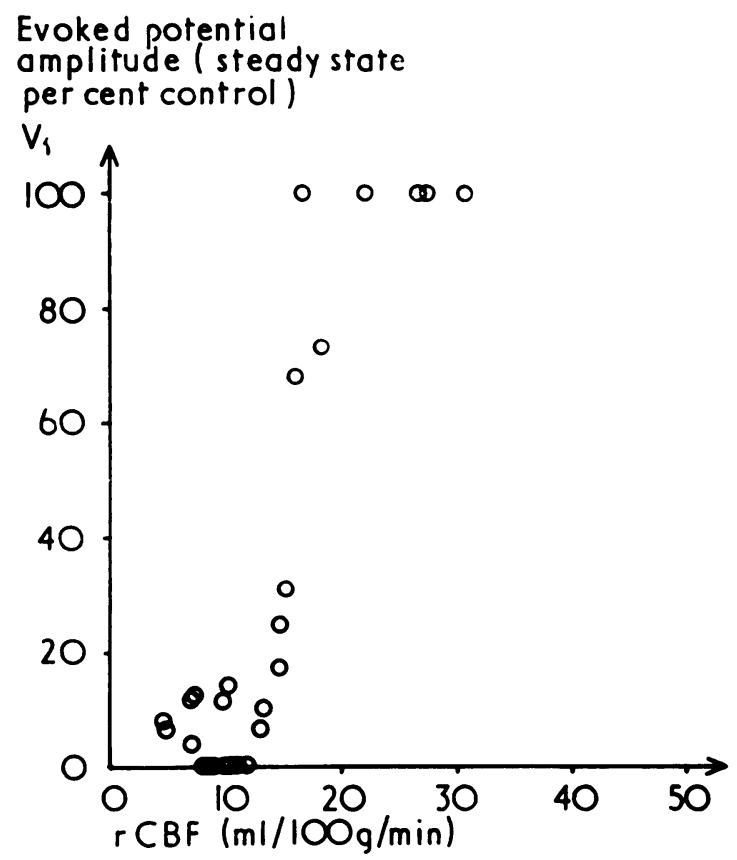

Fig 2 The relationship between amplitude of somatosensory evoked response after middle cerebral artery occlusion in the baboon and cortical blood flow in $\mathrm{ml} / 100 \mathrm{~g} / \mathrm{min}$.

relationship between flattening of the EEG and carotid perfusion during endarterectomy. They confirmed a fairly sharp threshold relationship at levels of flow similar to those of our experiments. A further interesting facet which emerged from our own study was that where residual blood flow at the recording electrode site was less than about $15 \mathrm{ml} /$ $100 \mathrm{~g} / \mathrm{min}$ the evoked potential amplitude diminished steadily until it became zero. The rate at which this depression of the evoked potential occurred, expressed as units of rate of change, was highly and signifi- 
cantly correlated with the residual blood flow $(r=0.5$, $\mathbf{P}<0.001$ ). This indicates a linear relationship between the rate of depression and the ultimate degree of ischaemia. We feel therefore that failure of neuronal function can be predicted in primate cortex where the local blood flow falls to below about 16 $\mathrm{ml} / 100 \mathrm{~g} / \mathrm{min}$.

\section{EVIDENCE FOR MAINTAINED STRUCTURE WITH LOST FUNCTION}

If we accept that loss of cortical function in the baboon is related to blood flows of less than $16 \mathrm{ml} /$ $100 \mathrm{~g} / \mathrm{min}$, then it is particularly interesting to note that the area of cortex which is involved in such a degree of ischaemia, that is, sufficient to abolish function, is very much wider than the area of cortex which proceeds to infarction. A group of comparable animals was maintained over three years while the characteristics of their clinical stroke were studied, and in a terminal experiment the characteristics of blood flow were assessed over the hemisphere affected by the infarct. The animals were then subjected to perfusion fixation of the brain with a formol-aceticacid-methanol-solution. Detailed histological studies were performed by Dr J. B. Brierley, whose findings have been reported (Brierley and Symon, 1977) and will be published in detail. While the area of cortex which one presumes to have lost function as evidenced by reduction of flow below the functional threshold is quite wide, the ultimate area of infarction is confined to areas where blood flow reduction in the acute stage of the stroke is a great deal more intense,

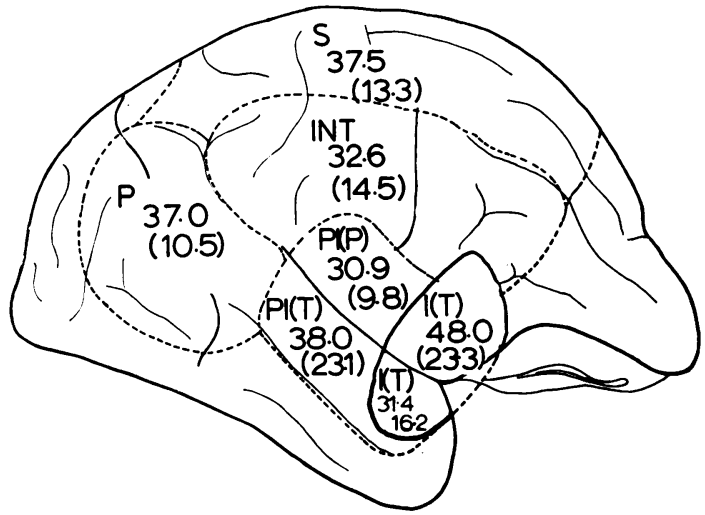

Fig 3 Cortical blood flow in $\mathrm{ml} / 100 \mathrm{~g} / \mathrm{min}$ three years following middle cerebral occlusion. $I(t)$ represents infarct blood flow, and the extent of the infarct is shown by a solid line surrounding these values. $P I(P)$ and $(t)$ represent periinfarct parietal and temporal, structurally normal but showing disordered vaso reactivity. INT represents an intermediate zone, $S$ and $P$ superior and posterior zone. Figures represent averaged blood flow over eight animals. and certainly below values of $10 \mathrm{ml} / 100 \mathrm{~g} / \mathrm{min}$ (fig 3). Together these two observations indicate that in the acute stage of infarction, loss of function willo affect neurones in a much wider distribution than the ultimate infarct.

EVIDENCE FOR THRESHOLD SENSITIVITY IN CELL MEMBRANE FUNCTION IN RELATION TO ISCHAEMIA

The development of potassium-sensitive micro- ${ }^{\infty}$ electrodes by Walker (1971) and their modification. $\vec{\circ}$ by Astrup and our own group (Astrup et al, 1977), $\overrightarrow{\vec{H}}$ allowed of the determination of movements of extracellular potassium during ischaemia, andत̄ revealed interesting relationships between the degree is of ischaemia and the extent of efflux of potassium into the extracellular space. Basic techniques were similar, the potassium micro-electrodes being intro- $\overrightarrow{-}$ duced at selected points in relation to blood flow $\vec{\theta}$ electrodes and to the evoked response electrode while ${ }_{\circ}$ the cortex was subjected to ischaemia of varying? density by middle cerebral occlusion and superadded $\vec{C}$ exsanguination. Potassium has been known to accummulate in the extracellular space during hypoxia (Collewjin and Schadé, 1962; Meyer et al, 1962; Vyskočil et al, 1972; Kirshner et al, 1976) aard $\overrightarrow{0}$ we were interested to determine both from the. $\checkmark$ pathological and clinical standpoint whether the accummulation of potassium in the extracellulars space accompanied the already demonstrated loss of $\frac{\vec{D}}{\bar{O}}$ the electrical activity or whether these two aspects of functional failure were differentially sensitive to $\stackrel{\mathbb{Q}}{\mathbb{Q}}$ ischaemia. Control levels of extracellular potassium $\overrightarrow{\vec{F}}$ ranged from 3 to 9 mmol (mean 5.7, SD 1.5) and $\frac{0}{3}$ increases in extracellular potassium associated with $\overline{-}$ reduction in flow followed two distinct types of timeo course (fig 4). The first was a slight but definite slow increase in extracellular potassium which tended to normalize over a period of 30 to $60 \mathrm{~min}$. The second $\frac{0}{3}$ type was a more massive increase in extracellular potassium (to levels of 30 to $80 \mathrm{mmol}$ ), and where we were able to determine upper and lower levels of flow between this massive extracellular potassium ${ }^{\circ}$ rise commences with certainty and to compare it with $\frac{}{O}$ the upper and lower flow level bracketing the disappearance of the evoked response, we found that $\tilde{\sigma}^{-}$ the massive increase in extracellular potassium $N$ always occurred at a lower level of tissue flow than failure of the evoked response. The levels for massive ${ }^{\omega}$ movements of potassium were between an upper flow bound of $11.4 \mathrm{ml} / 100 \mathrm{~g} / \mathrm{min}$ (SD 2.6) and a mearfe lower flow bound of $7.6 \mathrm{ml} / 100 \mathrm{~g} / \mathrm{min}$ (SD 2.2). Comparing the upper bound of the potassium shift with the lower bound of the evoked response failure there was a significant difference $(P<0.01)$. 


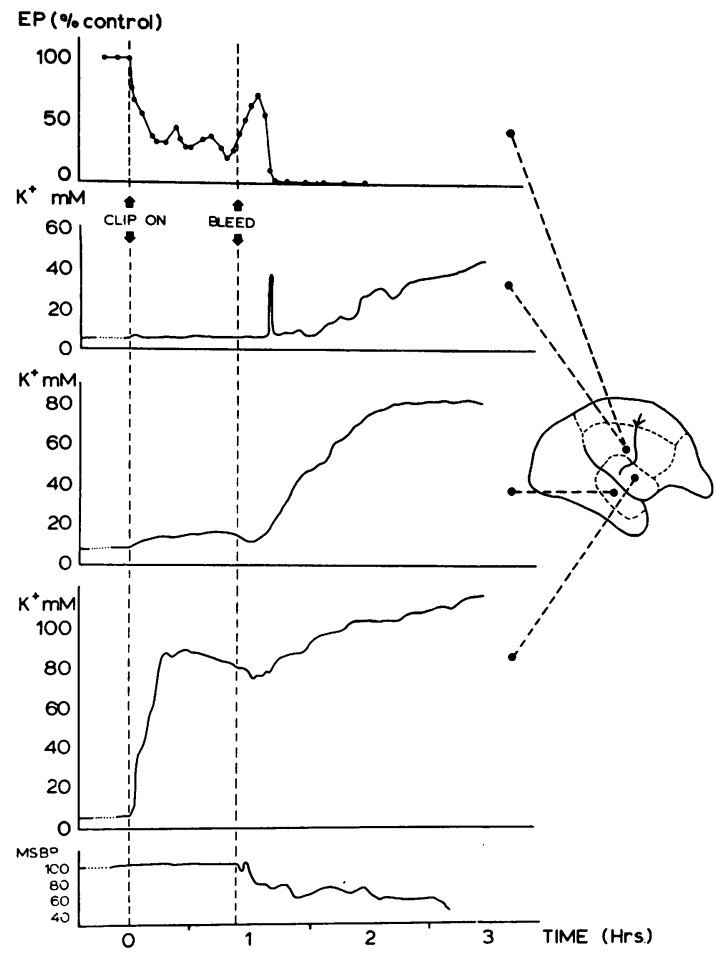

Fig 4 Reduction of amplitude of somatosensory evoked response and movements of potassium into the extracellular space induced by middle cerebral artery occlusion followed subsequently by reduction of the systemic blood pressure. The three potassium electrodes show, from above downwards, no effect of acute ischaemia, a moderate spontaneously resolving rise to $15 \mathrm{mM}$ and a massive rise in the area of severe ischaemia.

These findings confirmed an earlier joint study (Astrup et al, 1977) which had already contrasted the separation of potassium movement and electrical failure observed in our circumstances of graded ischaemia with the sudden loss of electrical activity and massive extracellular potassium movement demonstrated in experimental generalized hypoxia. It is clear that electrical failure occurs at a higher flow threshold, between 14 and $16 \mathrm{ml} / 100 \mathrm{~g} / \mathrm{min}$, than the massive rise in extracellular potassium, between 8 and $11 \mathrm{ml} / 100 \mathrm{~g} / \mathrm{min}$. The smaller self-limiting increases in potassium which we have observed are distinct from the massive rises, and do occur at flow levels similar to those at which electrical failure takes place.

The relationship between extracellular potassium movement and ischaemia is of course a complex one. It depends not only upon the failure of mechanisms of ionic pumping within cells so that potassium escapes to the outside, but is also influenced by the effects of ischaemia on normal methods of clearance of potassium from the extracellular space. These include active transport mechanisms (Hertz, 1966; Orkand, Nicholls, and Kuffler, 1966; Pollen and Trachtenberg, 1970; Haljamäe and Hamberger, 1971; Lewis and Schuette, 1975; Lothman et al, 1975), and also clearance of potassium through blood vessels. Both of these mechanisms are probably impaired by ischaemia and our experiments have not indicated the relative significance of impairment of each particular mechanism, but it seems certain that the rapidity and extent of the appearance of potassium in the extracellular space in relation to ischaemia below levels of $11 \mathrm{ml} / 100 \mathrm{~g} / \mathrm{min}$ must be related primarily to failure of membrane function. There is some evidence from a study of the rate of disappearance of potassium, either in reperfusion or in relation to spontaneous increases of potassium observed at levels of flow above those associated with membrane failure, that the rate of clearance of potassium bears a linear relationship to flow, and that potassium clearance from the extracellular space may well fail altogether at levels of flow in the region of 5 to $6 \mathrm{ml} / 100 \mathrm{~g} / \mathrm{min}$. While we cannot be clear cut about the level of flow associated with infarction, the distribution and density maps suggest that a level of flow appreciably below $10 \mathrm{ml} / 100 \mathrm{~g} / \mathrm{min}$ will be required, and it may well be that infarction of tissue requires degrees of ischaemia so profound as to interfere with clearance of ions from the extracellular space even by diffusion into the limited residual circulation.

Other evidence of a relationship between neuronal function and ischaemia

In collaboration with the neurochemical group of Professor Alan Davison and Dr Bowen (Bowen et $a l, 1976)$, we have attempted to link the degree of ischaemia to which cortex has been subjected with the function of subcellular components such as neurotransmitter uptake by synaptosomes. Thus, we have evidence that the synaptosomal uptake of the neurotransmitter GABA shows a linear decline in relation to flow, and, although the upper bound for this has not been defined with certainty, it appears likely that it is rather higher than that required to interfere with electrical function. Cholinergic neurones, however, show no such sensitivity, uptake remaining unimpaired to very much lower levels. The clear relationship between energy transport mechanisms and hypoxia has been extensively investigated by Siesjö and his group in Scandinavia (Siesjö and Nilsson, 1971) and by an extension of these techniques in our own and other groups, it is likely that a clear picture of the differential sensitivity 
of various facets of neuronal function to reduction in blood flow will be available within the next few years.

In general there is now good evidence that in an ischaemic process in the primate brain failure of function of neurones extends much more widely than ultimate infarction. This principle contains a profound therapeutic implication. In the first place it probably explains the gradual recovery of function which we observe in human ischaemic processes as flow rises above the threshold for restoration of function in neurones which have remained intact, though inactive. Secondly, it provides a powerful clinical stimulus to the development of techniques whereby the 'grey zone' of failure of function surrounding the structureless zone of complete infarction may be adequately reperfused and function resorted as a result.

\section{References}

Abbie, A. A. (1934). The morphology of the fore-brain arteries, with especial reference to the evolution of the basal ganglia. Journal of Anatomy, 68, 433-470.

Astrup, J., Symon, L., Branston, N. M., and Lassen, N. A. (1977). Cortical evoked potential and extracellular $\mathrm{K}^{+}$and $\mathrm{H}^{+}$at critical levels of brain ischamia. Stroke, in press.

Bowen, M. D., Goodhardt, M. J., Strong, A. J., Smith, S. B., White, P., Branston, N. M., Symon, L., and Davison, A. N. (1976). Biochemical indices of brain structure, function and "hypoxia" in cortex from baboons with middle cerebral artery occlusion. Brain Research, 117, 503-507.

Boyd, R. J., and Connolly, J. E. (1962). Tolerance of anoxia of the dog's brain at various temperatures. Surgical Forum, 12, 408-410.

Boysen, G., Engell, H. C., and Trojaborg, W. (1973). Effect of mechanical rCBF reduction on EEG in man. Stroke, 4, 361.

Branston, N. M., Symon, L., Crockard, H. A., and Pasztor, E. (1974). Relationship between the cortical evoked potential and local cortical blood flow following acute middle cerebral artery occlusion in the baboon. Experimental Neurology, 45, 195-208.

Brierley, J. B., and Symon, L. (1977). The extent of infarcts in baboon brains three years after division of the middle cerebral artery. Journal of Neuropathology and Applied Neurobiology, in press.

Collewjin, H., and Schadé, J. P. (1962). Potassium movements in the cerebral cortex during asphyxia. Acta Morphologica Neerlando-Scandinavica, 5, 11-20.

Dennis, C., and Kabat, H. (1939). Behaviour of dogs after complete temporary arrest of the cephalic circulation. Proceedings of the Society for Experimental Biology and Medicine, 40, 559-561.

Grenell, R. G. (1946). Central nervous system resistance. I. The effects of temporary arrest of cerebral circulation for a period of two to ten minutes. Journal of Neuropathology and Experimental Neurology, 5, 131-154.

Haljamäe, H., and Hamberger, A. (1971). Potassium accumulation by bulk prepared neuronal and glial cells. Journal of Neurochemistry, 18, 1903-1912.

Harvey, J., and Rasmussen, T. (1951). Occlusion of the middle cerebral artery: an experimental study. A.M.A. Archives of Neurology and Psychiatry, 66, 20-29.

Hertz, L. (1966). Neuroglial localization of potassium and sodium effects on respiration in brain. Journal of Neurochemistry, 13, 1373-1387.
Kirshner, H. S., Blank, W. F., Jr, and Myers, R. E. (1976) Changes in cortical subarachnoid fluid potassium concenev trations during hypoxia. Archives of Neurology, 33, 84-90

Lewis, D. V., and Schuette, W. H. (1975). NADH fluores cence and $\left[\mathrm{K}^{+}\right]_{0}$ changes during hippocampal electrica stimulation. Journal of Neurophysiology, 38, 405-417.

Lothman, E., LaManna, J., Cordingley, G., Rosenthal, M.ㅁ and Somjen, G. (1975). Responses of electrical potentialo potassium levels, and oxidative metabolic activity of the cerebral neocortex of cats. Brain Research, 88, 15-36.

Meyer, J. S., Gotoh, F., Tazaki, Y., Hamaguchi, K., Ishikawaฉ̊ S., Nouailhat, F., and Symon, L. (1962). Regional cerebraw blood flow and metabolism in vivo: effects of anoxia, hypoglycemia, ischemia, acidosis, alkalosis, and alterations of blood $\mathrm{pCO}_{2}$. Archives of Neurology, 7, 560-581.

Orkand, R. K., Nicholls, J. G., and Kuffler, S. W. (1966) $\overrightarrow{\vec{\omega}}$ Effect of nerve impulses on the membrane potential of gliap cells in the central nervous system amphibia. Journal of Neurophysiology, 29, 788-806.

Pasztor, E., Symon, L., Dorsch, N. W. C., and Branston, NW M. (1973). The hydrogen clearance method in assessment of blood flow in cortex, white matter and deep nuclei of baboons. Stroke, 4, 556-567.

Pollen, D. A., and Trachtenberg, M. C. (1970). Neuroglia $\overrightarrow{\vec{A}}$ gliosis and focal epilepsy. Science, 167, 1252-1253.

Shellshear, J. L. (1921). The basal arteries of the forebrain and their functional significance. Journal of Anatomy, 55, 27-35.

Siesjö, B. K., and Nilsson, L. (1971). The influence of arteriaร̆ hypoxemia upon labile phosphates and upon extracellula and intracellular lactate and pyruvate concentrations in the rat brain. Scandanavian Journal of Clinical and Laboratory Investigation, 27, 83-96.

Sundt, T. M., Jr, and Waltz, A. G. (1966). Experime\{̧ cerebral infarction: retro-orbital extra-dural approach fo occluding the middle cerebral artery. Proceedings of Mayo Clinic, 41, 159-168.

Symon, L. (1961). Studies of leptomeningeal collatera circulation in Macacus rhesus. Journal of Physiology, 159 68-86.

Symon, L. (1975). Experimental model of stroke in the baboon. Advances in Neurology, 10, 199-212.

Symon, I.., Branston, N. M., and Strong, A. J. (1976). Autoregulation in acute focal ischaemia. Stroke, 7, 547.

Symon, L., Dorsch, N. W. C., and Crockard, H. A. (1975) The production and clinical features of a chronic stroke. model in experimental primates. Stroke, 6, 476-481.

Symon, L., Khodadad, M. D., and Montoya, G. (1971). Theㅇำ effect of carbon dioxide inhalation on the pattern of gaseous metabolism in ischaemic zones of the primate. cortex. "An experimental study of the intracerebral steal' phenomenon in baboons. (In preparation.)

Symon, L., Pasztor, E., Dorsch, N. W. C., and Branston, N. M. (1973). Physiological responses of local areas of the을 cerebral circulation in experimental primates determined by the method of hydrogen clearance. Stroke, 4, 632-642.

Thompson, R. K., and Smith, G. W. (1951). Experimentalocclusion of the middle cerebral artery during arterial $N$ hypotension. Transactions of the American Neurologicat Association, 76, 203-207.

Vyskočil, F., Kriz, N., and Bures, J. (1972). Potassium-N selective microelectrodes used for measuring the extracel- $\omega$ lular brain potassium during spreading depression and anoxic depolarisation in rats. Brain Research, 39, 255-25960

Walker, J. L., Jr. (1971). Ion specific, liquid ion exchanger microelectrodes. Analytical Chemistry, 43, 83A-93A.

Weinberger, L. M., Gibbon, M. H., and Gibbon, J. H., Jr.: (1940). Temporary arrest of the circulation to the central nervous system. 2. Pathologic effects. Archives of Neurology and Psychiatry, 43, 961-986. 